A.I. Grabchenko, D.V. Romashov, D.O. Fedorenko, A.G. Mamalis, D. Lagoudas, V.A. Fedorovich and T. Baxevanis

\title{
Simulation of the effect of sintering on the integrity of diamond grains in grinding wheels
}

ABSTRACT. This paper focuses on theoretical studies of the process of manufacturing diamond abrasive tools and of diamond grinding through 3D modelling of the stress-strain state of the components of the diamond layer of the grinding wheel. Modelling of the sintering process of diamond grinding wheels with metal-based binders is used in order to determine the conditions needed to maintain the integrity of the diamond grains in the diamond layer.

Keywords: binder, destructive stress, diamond grains, finite element method, metal inclusion, sintering

Nanotechnology Perceptions 10 (2014) 42-53 